\title{
Symmetric Spaces of Exceptional Groups
}

\author{
Luis J. Boya ${ }^{* \dagger}$ \\ Departamento de Física Teórica \\ Universidad de Zaragoza \\ E-50009 Zaragoza \\ SPAIN
}

\begin{abstract}
We adress the problem of the reasons for the existence of 12 symmetric spaces with the exceptional Lie groups. The $1+2$ cases for $G_{2}$ and $F_{4}$ respectively are easily explained from the octonionic nature of these groups. The $4+3+2$ cases on the $E_{6,7,8}$ series require the magic square of Freudenthal and, for the split case, an appeal to the supergravity chain in 5,4 and 3 spacetime dimensions.
\end{abstract}

\section{Introduction}

Symmetric Spaces were introduced and classified by E. Cartan in 1926. They are riemannian spaces with an extra symmetry condition, namely geodesic reflection being isometry; they turn out to be homogeneous spaces $S=G / K$. This has as consequence that curvature is covariant constant, $\nabla R=0$; that fact can be used as an alternative definition. Here we shall refer mainly to the compact subclass; the standard source is the book by Helgason [1. From the Lie algebra $L=L(G)$ point of view, there is an involutory automorphism with $L(K)$ as the fix point set. Then if $P$ is a suplementary space, so $L(G)=L(K)+P$, $[L(K), L(K)]=L(K),[L(K), P]=P$, and $[P, P]=L(K)$. A good recent reference for physicists in this context is [2]. If $G$ and $K$ are compact, with Weyl's unitary trick $P \rightarrow i P$ we obtain the noncompact families of symmetric spaces.

There are seven families of symmetric spaces associated to the three division algebras, the reals $R$, the complex $C$ and the quaternions $H$, as the transitive groups ( $G$ above) in general are $O(n), U(n)$ and $S q(n)$, and there are also twelve single cases. The aim of this work is to understand the 12 exceptions as extensions of the classical families to the

\footnotetext{
*luisjo@unizar.es

${ }^{\dagger}$ To be published in the Proceedings of the $27^{\text {th }}$ International Conference on Group-Theoretical Methods in Physics. Yerevan, Armenia (2008).
} 
octonion algebra $O$.

This study must be seen as a case of understanding exceptional cases in mathematics: once one has classified objects, as e.g. in regular and exceptional classes, the question arises to place exceptions in some context, to see why they are there. One can ask, for example, why there are exceptional Lie groups (like $E_{8}$ ), or sporadic (finite simple) groups (like the Monster group), or why some regular polytopes exist in four dimensions only (like the 24-cell). Indeed, our problem has relation with other problems in Lie group theory: what are the real forms of (complex) Lie groups (classical or exceptional), which groups act transitively on spheres, and also with Berger's classification of the special holonomy manifolds; in this late case, for example, there are, besides the $3 \cdot 2=6$ families, for the groups $S O, O ; S U, U ; S q$ and $q(n):=[S q(n) \times S q(1)] / Z_{2}$, also two exceptions, again related to octonions, namely $G_{2}$ and $\operatorname{Spin}(7)$.

It is of course the lack of associativity of the octonion division algebra which makes the corresponding "series" finite in number.

So we are adressing a purely mathematical question. We hope, however, that the issue would be relevant for physics: exceptional groups appear conspicuously e.g. in string or $M$-Theory [3], and on general grounds one expects Nature to be described by a singular, non-generic mathematical structure, as Nature is unique (!). There are also recent applications of symmetric spaces to integrable systems and to random matrices. We shall make use of Supergravity in relation with symmetric spaces at the end of this report.

\section{Classification of Compact Symmetric Spaces}

The conventional (generic) symmetric spaces are very easy to describe. Let us write the series of groups and inclusions for the Orthogonal $(O)$, Unitary $(U)$ and compact Symplectic $(S q)$ series:

$$
O(n) \subset U(n) \subset S q(n) \subset U(2 n=m) \subset O(2 m)
$$

Roughly speaking, the four first classes of generic compact riemannian symmetric spaces are $U / O, S q / U, U(2) / S q$ and $O(2) / U$.

NOTE: For us $S q(n)$ is the compact form of the $C_{n}$ series of Cartan's list of simple Lie groups; we shall use also (as above) the notation $q(n)$ for the "nonunimodular" form of $S q(n)$. These should be understood as the "unitary" groups with quaternion entries, or with (double) unitary complex entries fixing an (extra) conjugation; "q" is for quaternion, "Sq" for unimodular quaternion, of course. 
The first four uniparametric families of (compact) symmetric spaces are just the consecutive quotients above:

$$
\begin{gathered}
S U(n) / S O(n), \quad S q(n) / U(n), \quad S U(2 n) / S q(n), \quad \text { and } S O(2 n) / U(n) . \\
\operatorname{Dim}(n-1)(n+2) / 2, \quad n(n+1), \quad(2 n+1)(n-1), \text { and } n(n-1) . \\
\text { Cartan notation: } A I, \quad C I, \quad A I I, \text { and DIII. }
\end{gathered}
$$

Besides, there are three biparametric families, with the same field, giving the grassmannians or collection of subspaces, namely $K(p+q) / K(p) \times K(q)$, where $K:=R, C, H$ or groups $O, U$ and $S q$ :

$$
S O(p+q) / S O(p) \times S O(q), \quad S U(p+q) / S U(p) \times S U(q), \quad S q(p+q) / S q(p) \times S q(q) .
$$

with dimensions $p q, 2 p q$ and $4 p q$ respectively. In Cartan's notation they are BDI, AIII and $C I I$.

These grasmannians are, as said, collection of subspaces; in particular, the important class of projective spaces are reported here, as e.g.

$$
R P^{n}=O(n+1) / O(n) \times O(1)=S O(n+1) / O(n)=S^{n} / S^{0}
$$

Similarly

$$
C P^{n}=\ldots=S U(n+1) / U(n)=S^{2 n-1} / S^{1}, \quad H P^{n}=\ldots=S q(n+1) / q(n)=S^{4 n-1} / S^{3}
$$

The Lie groups themselves are also symmetric spaces, because one can write $G=$ $[G \times G] / G_{\text {diag }}$. But we do not consider them further.

Symmetric spaces have also a rank, the dimension of the maximal totally geodesic flat submanifold. It corresponds to the abelian (Cartan) subalgebra of the Lie algebra of simple Lie groups. Symmetric spaces of rank one are, for example, the projective spaces and the spheres, e.g. $S^{n}=S O(n+1) / S O(n)$ and $S^{2 n-1}=S U(n) / S U(n-1)$, as $S O(1)=S U(1)=1$. These spaces are also called two-point homogeneous spaces, Cfr. [1]. The free classical motion on them is superintegrable, maximally antiergodic (the orbits filling up just a curve (or $1 d$ manifold)), and quantization yields a discrete spectrum, depending on a single quantum number.

There remain the twelve exceptional symmetric spaces, $S=G / H$, where $G$ is one of the five exceptional groups. Cartan found the $1+2+4+3+2=12$ symmetric spaces associated respectively to the exceptional groups $G_{2}, F_{4}, E_{6}, E_{7}$ and $E_{8}$. To understand them, firts we have to see precisely the relation they have with the octonion division algebra. 


\section{Generalities on Octonions and Related Groups}

We start from the reals $R$; it is an ordered field, $x<y$ makes sense, and there are no automorphims, $\operatorname{Aut}(R)=1$. Now in $R^{2}$ we define an $R$-algebra by $i^{2}=-1$, where $i=(0,1)$. Then the complex numbers $\mathrm{C}$ are $z=x+i y$ (Gauss). Conjugation, norm and inverse are given as $\bar{z}=x-i y, N(z)=\bar{z} z$, and for $z \neq 0, z^{-1}=\frac{\bar{z}}{N(z)}$. Conjugation is the only continuous automorphism, $\operatorname{Aut}_{c}(C)=Z_{2}$; the field $\mathrm{C}$ is no longer ordered; both $R$ and $C$ are composition algebras, meaning the norm verifies $N\left(z_{1} z_{2}\right)=N\left(z_{1}\right) N\left(z_{2}\right)$. We have the inclusions $R^{2} \supset S^{1} \supset S^{0}$ for all complex numbers $C$, the unit complex numbers, and the imaginary units $\pm i$.

For the quaternions $H$ we proceed similarly (Hamilton, 1842). In $R^{4}$ define $i=$ $(0,1,0,0), j=(0,0,1,0)$ and $k=i j=(0,0,0,1)$ with $i^{2}=j^{2}=k^{2}=-1$, which requires $i j+j i=0$. Conjugation, norm and inverse are defined likewise as $q:=u+i x+j y+k z \equiv$ $\operatorname{Re}(q)+\operatorname{Im}(q)$ and then $\bar{q}=\operatorname{Re}(q)-\operatorname{Im}(q), N(q)=\bar{q} q$, and for $q \neq 0, q^{-1}=\frac{\bar{q}}{N(q)}$. The inclusions now are $R^{4} \supset S^{3} \supset S^{2}$ for all quaternions $H$, the unit quaternions and the imaginary units. Conjugation now is antiautomorphism, but arbitrary rotations of the imaginary units are the automorphims, $\operatorname{Aut}(H)=S O(3) . H$ is an anticommutative (skew-)field. Note $N\left(q_{1} q_{2}\right)=\overline{q_{1} q_{2}} q_{1} q_{2}=\overline{q_{2}} \overline{q_{1}} q_{1} q_{2}=N\left(q_{1}\right) N\left(q_{2}\right)$

For the octonions $O$ we proceed similarly (Greaves, 1842; Cayley, 1845). In $R^{8}$ define three independent antiinvolutory anticommutative units $i, j, k$ (e.g. with $i^{2}=j^{2}=k^{2}=$ $-1, i j+j i=0$ etc). Now force the same properties for the products $i j, j k, k i ;(i j) k$ : this implies nonassociativity, namely $(i j) k=-i(j k)$, the most remarkable (for unusual) property of octonions. There are $1+\left(\begin{array}{l}3 \\ 1\end{array}\right)+\left(\begin{array}{l}3 \\ 2\end{array}\right)+1=8$ units, seven imaginary. Conjugation, norm and inverse are defined likewise; the automorphim group should be a subgroup of $S O(7)$ : the inclusions now are $R^{8} \supset S^{7} \supset S^{6}$. So $\operatorname{Aut}(O)$ is $G_{2}$, the first (smallest) exceptional simple Lie group, of dimension 14 and rank 2. The octonions form a composition division noncommutative nonassociative algebra; in particular $N\left(o_{1} o_{2}\right)=N\left(o_{1}\right) N\left(o_{2}\right)$ still holds. The concrete expression $(i j) k=-i(j k)$ is called alternativity, and does characterize the octonions: in general $\left[\mathrm{O}_{1} \mathrm{O}_{2} \mathrm{O}_{3}\right]:=\left(\mathrm{O}_{1} \mathrm{O}_{2}\right) \mathrm{O}_{3}-\mathrm{O}_{1}\left(\mathrm{O}_{2} \mathrm{O}_{3}\right)$ is fully antisymmetric. For a further study, consult [4].

For the reals $R$ we have the units $S^{0}=( \pm 1)=Z_{2}=O(1)$. For the complex $C$ the units are $S^{1}=\exp (\mathrm{i} \phi)=\mathrm{SO}(2)=\mathrm{U}(1)$. For the quaternions $H$ we have $S^{3}=\operatorname{Spin}(3)=\mathrm{SU}(2)=$ $\mathrm{Sq}(1)$. But for the octonions $O$ the sphere $S^{7}$ is not a group, only a parallelizable manifold (i.e. the tangent bundle is trivial). However, as shown by Ramond [5], the seven sphere becomes a group after stabilization by $G_{2}=\operatorname{Aut}(\mathrm{O})$, and becomes the group $\operatorname{Spin}(7)$ !

$$
\operatorname{Spin}(7) \sim \mathrm{G}_{2} \vee \mathrm{S}^{7}
$$

We need now some results from Lie group theory. Firts, any compact simple Lie group can be understood (Hopf, ca. 1940) as finitely twisted product of odd-dimensional spheres, 
symbolically

$$
G \sim \Pi_{j} \odot S^{2 j+1}
$$

where $j$ are called the exponents; for all this see [6]. For example. $S U(2)=S^{3}$ is known, $\operatorname{Spin}(4)=\mathrm{S}^{3} \times \mathrm{S}^{3}$ is true, but also $S U(3) \sim S^{3} \odot S^{5}$, etc. Other result is that the exponents are palindromic (Kostant), i.e., the differences left-right are the same. For example, the exponents $j$ for $\operatorname{Spin}(10)$ are $(1,3,4,5,7)$.

Now we compare $S q(1)=S^{3}$ and $q(1)=S^{3} \times_{2} S^{3}$ with $G_{2} \sim S^{3} \times S^{11}$ and $\operatorname{Spin}(7) \sim$ $S^{3} \times S^{7} \times S^{11}$. We shall call

$$
S^{7} \sim \operatorname{Oct}(1)^{\prime}, \quad G_{2} \sim \operatorname{SOct}(1), \quad \operatorname{Spin}(7) \sim \operatorname{Oct}(1)
$$

on the understanding that ' Oct $(1)^{\prime}$ is NOT a group. To justify the notation, we remark: $S q(1) \rightarrow q(1) \rightarrow S^{3}$ corresponds to $\operatorname{SOct}(1) \rightarrow \operatorname{Oct}(1) \rightarrow S^{7}$ which is true: $\operatorname{Spin}(7) / G_{2}=S^{7}$. There are more examples, e.g. $S q(1)^{2}=S^{3} \times S^{3}$ corresponds to $\operatorname{Oct}(1)^{2}=S^{3} \odot S^{7} \odot S^{7} \odot S^{11}=\operatorname{Spin}(8)$. Now as $q(2) \sim S q(1) \times S q(2) \sim S^{3} \odot S^{3} \odot S^{7}$, it leads to $\operatorname{Oct}(2) \sim S^{3} \odot S^{7} \odot S^{11} \odot S^{15}=\operatorname{Spin}(9)$ !

We can go up to $n=3$, but there is a surprise! 'Oct $(3)^{\prime}$ would have exponents $j:(1,3,5,7,11)$, as corresponds to spheres $(7,15,23)$ stabilized by $(3,11)$. But this combination is NOT palindromic! However, it becomes so by losing the $S^{7}$ sphere $((1,5,7,11)$ is OK!). Losing the 7 -sphere is the unimodular ("S") restriction, and indeed we have concocted $F_{4}$ :

$$
F_{4} \sim S^{3} \odot S^{11} \odot S^{15} \odot S^{23}=\operatorname{SOct}(3)
$$

which is the second exceptional Lie group, with dimension $52=3+11+15+23$ and rank 4 (number of spheres)!

We stop here for the moment. As summary, we have "shown" the following "equivalences" 'Oct $(1)^{\prime}=S^{7}, G_{2}=\operatorname{Aut}(O) \sim S^{3} \odot S^{11}=S O c t(1), \operatorname{Oct}(1)=S^{3} \odot S^{7} \odot S^{11}=$ $\operatorname{Spin}(7), \operatorname{Oct}(1)^{2}=\operatorname{Spin}(8), \operatorname{Oct}(2)=\operatorname{Spin}(9), \operatorname{SOct}(3)=F_{4}$.

\section{Symmetric Spaces of Exceptional Groups: $G_{2}$ and $F_{4}$}

We are ready now no address the exceptional symmetric spaces. As we say that $G_{2}=$ $S O c t(1)$ and $\operatorname{Spin}(7)=\operatorname{Oct}(1)$, we generalize the $S q(1)$ case: its only symmetric space is of $q / U$ type, namely $S q(1) / U(1) \approx S^{3} / S^{1}=S^{2}=C P^{1}$. Hence the octonionic equivalent must be 


$$
\operatorname{SOct}(1) / q(1) \approx G_{2} / S O(4) \approx \frac{S^{3} \odot S^{11}}{S^{3} \odot S^{3}} \approx H P^{2}
$$

It is the only symmetric space with $G_{2}$. We do not care about the "Octonionic" forms for $\operatorname{Spin}(7,8,9)$, so our next case will be $F_{4}$. Recalling $F_{4}=\operatorname{SOct}(3)$, there are now two possibilities: $\operatorname{Oct} / S q$ and $\operatorname{Oct}(3) / \operatorname{Oct}(1) \times \operatorname{Oct}(2)$; both are present: from

$$
\begin{gathered}
O(2) \subset U(2) \subset q(2) \subset O c t(2)=\operatorname{Spin}(9) \\
O(3) \subset U(3) \subset q(3) \subset \operatorname{SOct}(3)=F_{4}
\end{gathered}
$$

we get $\operatorname{SOct}(3) / \operatorname{Oct}(2)=F_{4} / \operatorname{Spin}(9)=O P^{2}$, i.e., the Moufang (octonionic, projective) plane. But also $S O c t(3) / q(3)=F_{4} / S q(3) \times S q(1)$, the other symmetric $F_{4}$-space.

There is another case, namely $\operatorname{Oct}(2) / O c t(1)^{2} \approx \operatorname{Spin}(9) / \operatorname{Spin}(8)=S^{8}=O P^{1}$, but this is already accounted for, e.g. as $S^{8}=S O(9) / S O(8)$.

Are there more cases? One proves that all other putative cases have been already accounted for. For example, from $U(2) / U(1)=S^{3}$ and $q(2) / q(1)=S^{7}$, one would expect $\operatorname{Oct}(2) / O c t(1)=S^{15}$. Indeed $\operatorname{Spin}(9) / \operatorname{Spin}(7)=S O(9) / S O(7) \equiv S O(9) / S O(7) \times S O(1)$, dejà $v u$, as it is an orthogonal grassmannian (and $S^{15}$ plays the role of a Stiefel manifold); it corresponds to the principal bundle $\operatorname{Spin}(7) \rightarrow \operatorname{Spin}(9) \rightarrow S^{15}$, an exceptional transitive action on spheres: in fact, $\operatorname{Spin}(9)$ is the largest Spin group acting trans in any maximal sphere!

So we have $S=G_{2} / S O(4)$ with $\operatorname{dim} S=8$; it is Cartan's $G$ space. And $S^{\prime}=$ $F_{4} / \operatorname{Spin}(9)$ with dim 16 ; it is Cartan's FII space. Finally $S^{\prime \prime}=F_{4} / S q(3) \times S q(1)$ with $\operatorname{dim} 28$; it is Cartan's FI space.

\section{Magic Square and the E-Series: the Other Sym- metric Spaces}

For the $E$-series we anticipate our construction of the symmetric spaces is not so clearcut. One of the reasons is, undoubtedly, that our understanding of these groups is still incomplete, inspite the efforts of Freudenthal, Tits, and others. We shall resort to two contructions for these groups: (1), The Magic Square of Freudenthal, Rosenthal and Tits 
(1956), and (2) an appeal to physics, in the existence of the chain of maximal supergravities, from spacetime dimensions 11 to $5,4,3$.

Recall the inclusions already seen $O(n) \subset U(n) \subset q(n) \subset U(2 n) \subset O(4 n)$, which holds for any $n$; it might be "filled in" for $n=2$ and $n=3$ as follows:

$$
\begin{gathered}
O(2) U(2) S q(2) \operatorname{Oct}(2)=\operatorname{Spin}(9) \\
U(2) U(2)^{2} U(4) \operatorname{Oct}_{C}(2)=\operatorname{Spin}(10) \\
S q(2) U(4) O(8) \operatorname{Oct}_{H}(2)=\operatorname{Spin}(12) \\
\operatorname{Spin}(9) \operatorname{Spin}(10) \operatorname{Spin}(12) \operatorname{Spin}(16)=\text { Oct }_{O}(2)
\end{gathered}
$$

Also, the primordial Magic Square is

$$
\begin{aligned}
& O(3) U(3) S q(3) \operatorname{Oct}(2)=F_{4} \\
& U(3) U(3)^{2} U(6) E_{6} \\
& S q(3) U(6) O(12) E_{7} \\
& \begin{array}{lllll}
F_{4} & E_{6} & E_{7} & E_{8}
\end{array}
\end{aligned}
$$

The last row/column must be understood as $\operatorname{SOct}(3)=F_{4}$, extended by the complex $\left(E_{6}\right)$, the quaternions $\left(E_{7}\right)$ and the octonions $\left(E_{8}\right)$. Of course, the right way to understand these groups is as automorphism groups for the $3 \times 3$ hermitian Jordan algebras over the bi-rings octonions $\otimes$ reals $=$ octonions, octonions $\otimes$ complex, octonions $\otimes$ quaternions and octonions $\otimes$ octonions. See e.g. [8]

Now for $E_{6}$ : we have the three inclusions

$$
F_{4} \subset E_{6}, \quad U(6) \subset E_{6}, \quad O(10) \subset E_{6}
$$

which generates three of the 4 cases for $E_{6}$. For $E_{7}$ we have also the three inclusions

$$
E_{6} \subset E_{7}, \quad O(12) \subset E_{7} \text { (repeated!) }
$$

which generates two of the 3 cases for $E_{7}$. For $E_{8}$ we have also two more cases:

$$
E_{7} \subset E_{8}, \quad O(16) \subset E_{8} \text { (repeated!) }
$$

which generates the two cases for $E_{8}$.

The folowing eqs. summarizes the $3+2+2$ cases: 


$$
\begin{aligned}
E_{6} / F_{4}, E_{6} /(S U(6) \times S U(2)), & E_{6} / S O(10) \times U(1) E_{7} / E_{6} \times U(1), E_{7} / O(12) \times S q(1) ; \\
& E_{8} / E_{7} \times S q(1), E_{8} / O(16) .
\end{aligned}
$$

with dimensions $26,40,32 ; 54,64 ; 112,128$

corresponding to Cartan's EIV, EII, EIII; EVII, EVI; EIX and EVIII.

Before looking for the remaining two cases (one for each $E_{6}, E_{7}$ ) we pause to remark that the three following cases appear in physics:

$F_{4} / \operatorname{Spin}(9)=O P^{2}$ (M Theory), $E_{6} / \operatorname{Spin}(10) \times S O(2)$ (F Theory) $E_{8} / S O(16)$ (Superstrings)

The astute reader would have notices that the two remaining cases are the split form of the $E_{6,7}$ groups, namely the quotients by $S q(4)$ and $S U(8)$ bzw., which correspond to the split form divided by the maximal compact subgroup of the mother group. Where are they? First, recall the notations

$$
E_{6}^{\text {compact }}=E_{6(-78)}, E_{6}^{\text {split }}:=E_{6(+6)} \approx R^{42} \times S q(4)(\operatorname{dim} 36)
$$

We have no better way to understand the remaining symmetric spaces that to draw the sequences of groups and subgroups appearing in Supergravity, from dim 11 down to $\operatorname{dim} 3$, in such a way that the scalars form a sigma-like model. In fact, these pairs groupssubgroups are the split form:

$$
\begin{gathered}
E_{8} \supset E_{7} \supset E_{6} \supset D_{5} \supset A_{4} \supset \ldots \\
O(16) \supset S U(8) \supset S q(4) \supset S q(2)^{2} \supset S q(2) \supset \ldots
\end{gathered}
$$

which corresponds, in the descent from the 11-dim Supergravity of [7] to

3d, 128 scalars. $4 d, 35+35$ scalars. $5 d, 42$ scalars. $6 d, 25$ scalars, $7 d, 14$ scalars...

It happens that the subgroup sequence corresponds exactly to the maximal compact subgroup of the main group sequence (I do not know why this is so).

This gives us the remaining two symmetric spaces: for $E_{6}$ we have: $S q(4)$ with the traceless four-form makes up $E_{6}$, with $(36+42=78)$. For $E_{7}$ we have: $S U(8)$ with the four-form makes up $E_{7}$, as $63+70=133$. Finally, although we already obtained that, for $E_{8}$ we have: $S O(16)$ with the Spin irrep make up $E_{8}$, with $120+128=248$. In this connection see [9]. The first two were the missing exceptional symmetric spaces, corresponding 
to Cartan's $E I$, for $E_{6} / S q(4)$, and $E V$ for $E_{7} / S U(8)$.

Notice the sequences of groups $G(n), G(n)^{2}, G(2 n)$ and $O(n), U(n), S q(n)$ appear repeated many times. To quote a single example, the chain of first Spin groups is $\operatorname{Spin}(1)=$ $O(1), \operatorname{Spin}(2)=U(1), \operatorname{Spin}(3)=S q(1), \operatorname{Spin}(4)=S q(1)^{2}=q(1), \operatorname{Spin}(5)=\operatorname{Sq}(2), \operatorname{Spin}(6)=$ $S U(4)$, etc. It reminds one of Bott's periodicity.

\section{Conclusions}

1) The twelve exceptional symmetric spaces are obtained in a natural way, from pairs group/subgroup, both related to the octonions!

2) For $G_{2}$ and $F_{4}$, the arguments are unequivocal and unavoidable.

$3)$ For the $E$ series we present two arguments: one is the natural generalization of the inclusions $O \subset U \subset S q \subset O c t$, but the second argument makes use of a series of $E$-groups and subgroups for which the evidence is physical, not mathematical! This undoubtedly reflects the fact, already alluded to, that our understanding of the $E$ series is still incomplete.

4) During the writeup of our talk, the paper by Ferrara appeared [10], which exploit the relation among irreducible Riemannian globally symmetric spaces and supergravity theories in 3, 4 and 5 space-time dimensions, and therefore there is some overlap with our considerations on the last two exceptional symmetric spaces.

Acknowledgements. I thank the organizers of the $27^{\text {th }}$ ICGTMP meeting at Yerevan (Armenia), especially G. Pogosyan, for the very stimulating Conference. I thank the participants A. Solomon, F. Toppan and the belgian group (U. of Ghent) for discussions. 


\section{References}

[1] S. Helgason, Symmetric Spaces. Academic, 1962. Second enlarged edition, 1978.

[2] U. Magnea, An introduction to symmetric spaces, arXiv:cond-mat/0205288.

[3] L. J. Boya, Octonions and M-Theory. IOP Conf. Ser. 173 (2003) 245-248

[4] J. Baez, The Octonions. Bull. Am. Math. Soc. 39 (2001) 145-205.

[5] P. Ramond, Introduction to Exceptional Lie Groups and Algebras, Caltech Preprint CALT-68-577. December, 1976. It is regrettable that this important contribution of Pierre Ramond never appeared published.

[6] L. J. Boya, Rep. Math. Phys. 30 (1991) 149-162.

[7] E. Cremmer, B. Julia and J. Scherk, Phys. Lett. 76 (1978) 409-12.

[8] H. Freundenthal, Adv. Math. 1 (1964) 145-190.

[9] J. Adams, Lectures on Exceptional Lie Groups, U. Chicago 1978.

[10] S. Ferrara and A. Marrani, Symmetric Spaces in Supergravity, arXiv:0808.3567 [hepth] 